\title{
Sudoku Solving Ability and Intelligence
}

\author{
Onokpasa Eva \\ Department of Computer Science, \\ University of Jos
}

\author{
Bisandu Desmond \\ Department of Computer Science, \\ University of Jos
}

\author{
Ikoojo Simon \\ Department of Computer Science, \\ University of Jos
}

\begin{abstract}
Sudoku is a challenging, interesting and brain tasking game. Is there a correlation between human intelligence and human Sudoku solving ability? This research work uses as a case study, a class of third year computer science students of the University of Jos, to determine whether there exists a correlation between an individual's Sudoku solving ability and his intelligence.
\end{abstract}

\section{Keywords}

Sudoku, human intelligence, intelligence quotient and correlation.

\section{INTRODUCTION}

This research work is aimed at establishing whether or not a correlation exists between human intelligence and human Sudoku solving ability. Now, to achieve this it is important to clearly define and understand the definitions and measures of human intelligence. This will be discussed in the next section. In the methodology and evaluation sections, a comparison is made of the results of the performance of 29 university students in an IQ test as against their performance in solving 6 sudoku puzzles of varying levels of difficulties. In the concluding section a summary of the findings are stated.

\section{LITERATURE REVIEW}

"Intelligence is the ability to think, to learn from experience, to solve problems, and to adapt to new situations" [1]. The measure of human intelligence or cognitive ability is controversial. However this review focuses on what most psychologists have come to agree on with respect to human intelligence. Charles spearman called the construct that the different abilities and skills measured on intelligence tests have in common the general intelligence factor $(g)$ [2]. Most psychologists believe that people with higher $g$ learn faster. Next, a definition is given for intelligence quotient and some of the various IQ (Intelligence quotient) tests.

\subsection{Intelligence Quotient}

Intelligence quotient (IQ), is "a measure of intelligence that is adjusted for age" [3]. Below is the mathematical formula for IQ:
IQ $=$ mental age $\div$ chronological age $\times 100$.

A person's mental age is determined by his performance in Intelligence quotient tests. The following section consider some of these IQ tests

\subsection{Binet-Simon IQ test}

Alfred Binet, Victor Henri and Théodore Simon worked together to create the Binet-Simon test in 1905. This test was originally designed to reveal a child's mental age[4]. This test is designed by testing children of a specific age, e.g an 8 year old child would take an IQ test designed for 8 year olds, and this means that this test is designed such that most normal 8 year olds will have average scores on this test. However 8 year olds with learning disabilities will have lower scores and smarter 8 year olds will have higher scores.

\subsection{Stanford-Binet IQ test}

In 1916 Lewis M. Terman of Stanford university made an improvement to the Binet-Simon test, and this test is known as the Standford-Binet test.

\subsection{Wechsler Adult Intelligence Scale}

David Wechsler developed the first standardized adult intelligence test, the Bellevue-Wechsler Scale, in 1939, the Wechsler Intelligence Scale for Children, published in 1949 and revised in 1974, are acclaimed today as the best tests for measuring intelligence [5].

\section{METHODOLOGY}

In this research work a class of third year undergraduate students of Computer science at the University of Jos, were used as the case study. Their ages fall between the range of 20 - 25 years. There are 13 females 16 males. They were each handed 6 sudoku puzzles to solve within 72 hours. These puzzles were taken from [ $6 \&$ \& $]$. After retrieving their Sudoku solutions, they were given a 6 minute Wechsler Adult intelligence test available at [8]. Their Sudoku solutions were scored based on how many blank cells they filled in correctly divided by the total blank cells and multiplying the result by 100. The scores are summarized in table 1 .

\begin{tabular}{|c|c|c|}
\hline Student & Sudoku score (\%) & $\begin{array}{c}\text { Wechsler Adult Intelligence (WAIT) test } \\
\text { scores (\%) }\end{array}$ \\
\hline 1 & 42.03 & 40 \\
\hline 2 & 75.93 & 45 \\
\hline 3 & 54.24 & 50 \\
\hline 4 & 57.63 & 35 \\
\hline 5 & 63.39 & 55 \\
\hline 6 & 52.88 & 45 \\
\hline 7 & 64.41 & 50 \\
\hline
\end{tabular}




\begin{tabular}{|c|c|c|}
\hline 8 & 55.59 & 25 \\
\hline 9 & 67.46 & 55 \\
\hline 10 & 70.51 & 40 \\
\hline 11 & 74.92 & 30 \\
\hline 12 & 41.69 & 55 \\
\hline 13 & 57.97 & 45 \\
\hline 14 & 84.41 & 65 \\
\hline 15 & 81.69 & 45 \\
\hline 16 & 47.46 & 70 \\
\hline 17 & 79.66 & 75 \\
\hline 18 & 74.24 & 90 \\
\hline 19 & 85.08 & 90 \\
\hline 20 & 40.34 & 55 \\
\hline 21 & 28.47 & 50 \\
\hline 22 & 48.47 & 60 \\
\hline 23 & 25.42 & 35 \\
\hline 24 & 57.97 & 75 \\
\hline 25 & 44.07 & 60 \\
\hline 26 & 38.98 & 60 \\
\hline 27 & 36.27 & 35 \\
\hline 28 & 98.98 & 40 \\
\hline 29 & 91.53 & 20 \\
\hline
\end{tabular}

Table 1. Sudoku scores and Wechsler Adult Intelligence test scores obtained by 29 university undergraduate students

\section{DETERMINING AND EVALUATING THE DEGREE OF CORRELATION}

To determine graphically the degree of correlation between the Sudoku scores obtained and the WAIT scores a scatter plot

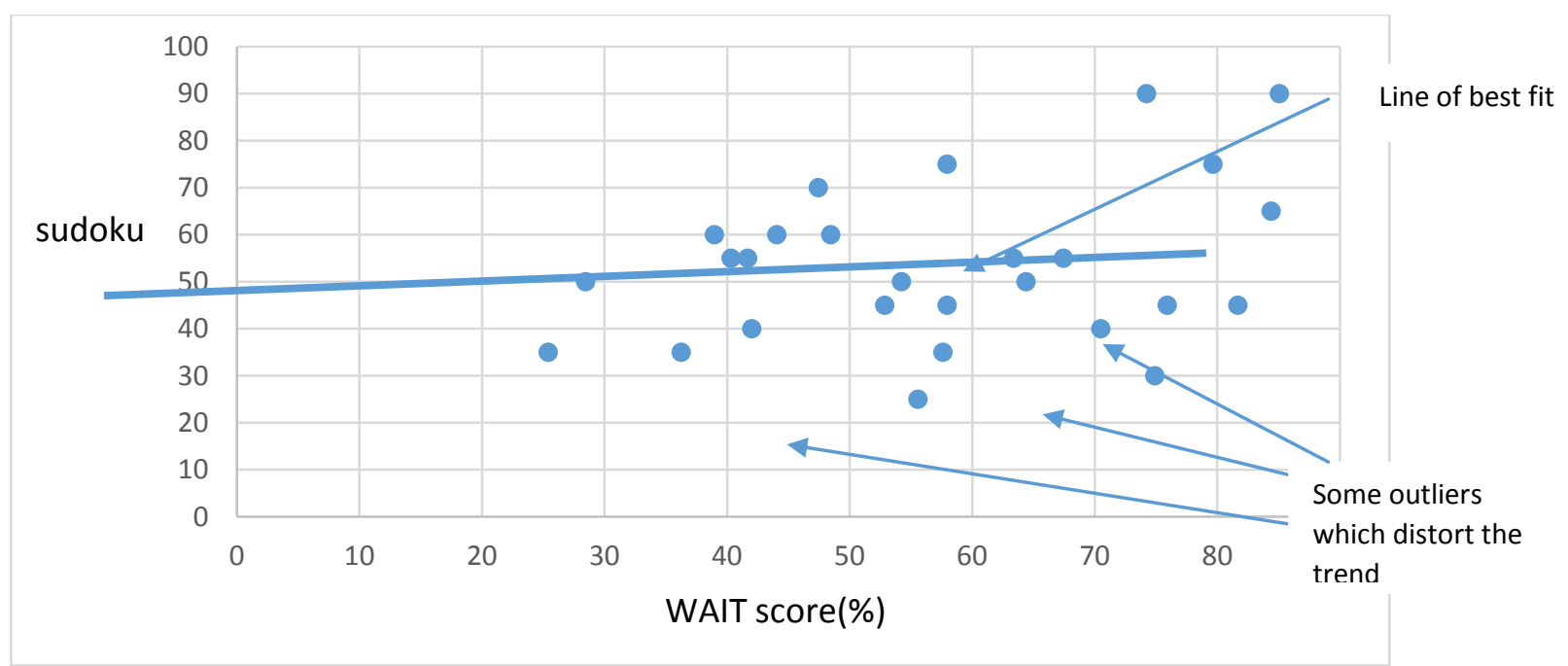

Fig 1. The scatter plot diagram shows each student's Sudoku score against their score in the Wechsler Adult Intelligence Test (WAIT) is made of the result displayed in table 1 . This is displayed in figure 1. 
Next, the correlation coefficient between the Sudoku scores and the WAIT score is calculated using the Pearson's correlation coefficient formula [9]:

$$
r=\frac{n\left(\sum x y\right)-\left(\sum x\right)\left(\sum y\right)}{\sqrt{\left[n \sum x^{2}-\left(\sum x\right)^{2}\right]\left[n \sum y^{2}-\left(\sum y\right)^{2}\right]}}
$$

$r=0.319752$

Where $x$ is the WAIT scores and $\mathrm{y}$ is the Sudoku scores and $\mathrm{n}$ the number of students used in this case study.

Since $\mathrm{r}$ ranges from 0 to 0.5 this implies a weak positive correlation between the Sudoku scores and the WAIT test scores. Also the scatter plot shown in fig 1 shows an uphill trend from left to right for majority of the points except for a few outliers, which distort the trend. Next the least square method is employed to determine the line of best fit.

The equation of a straight line is given by

$$
y=m x+c
$$

Where $\mathrm{m}=$ slope and $\mathrm{c}$ is the intercept with the $\mathrm{y}$-axis.

From the least square method [10]

$$
\begin{gathered}
m=\frac{n \sum(x y)-\sum x \sum y}{n \sum\left(x^{2}\right)-\left(\sum x\right)^{2}} \\
c=\frac{\sum y-m \sum x}{n}
\end{gathered}
$$

Applying the formulas above gives the values:

$$
\mathrm{m}=0.098091692, \mathrm{c}=55.0018
$$

with the values for $\mathrm{m}$ and $\mathrm{c}$ above a line of best fit is drawn as shown in fig 1 . This line has a very gentle positive slope, this indicates a very weak positive correlation.

\section{CONCLUSION AND FUTURE WORK}

From this case study it can be concluded that an individual who is skilled at solving Sudoku puzzles likely has a high general IQ. The results of the weak correlation between Sudoku scores and the WAIT test indicates that in some cases a high Sudoku doesn't necessarily mean a high general IQ. But this study was limited to a case. Would the result differ if a larger population of individuals were under studied? What if a specialized IQ test (say arithmetic or algebraic) was taken instead of a general IQ test, would the correlation between the Sudoku scores and IQ scores be stronger? These questions would be answered in future researches.

\section{REFERENCES}

[1] University of Minnesota (2018)Introduction to Psychology, Defining and Measuring Intelligencehttps://open.lib.umn.edu/intropsyc/chapter/91-defining-and-measuring-intelligence/

[2] Gottfredson, L. S. (1997). Mainstream science on intelligence: An editorial with 52 signatories, history and bibliography. Intelligence, 24(1), 13-23.

[3] Glossary of Important Assessment and Measurement Terms.(2016)Philadelphia, PA: National Council on Measurement in Education. 2016. intelligence quotient (IQ). Retrieved 2017-07-01.

[4] Kaufman, Alan S. (2009).IQ Testing 101. New York: Springer Publishing. ISBN 978-0-8261-0629-2. Lay summary(10 August 2010).

[5] Garraty J. \& Carnes, M. (1999)"Wechsler, David." In American national biography Vol 22. New York: Oxford University Press, 1999.

[6] www.sudoku.org.uk (2018)http://www.sudoku.org.uk/Daily.aspAccessed:20/ $03 / 2019$

[7] www.aisudoku.com (2006)http://www.aisudoku.com/index_en.htmlAccessed :20/03/2019

[8] free-iqtest (2018)http://www.free-iqtest.net[Accessed 01/01/2019]

[9] Pearson K. (1895) Notes on regression and inheritance in the case of two parents, Proceedings of the Royal Society of London, $58: 240-242$.

[10] Charnes, A., Frome, E. L.\& Yu, P. L. (1976). The Equivalence of Generalized Least Squares and Maximum Likelihood Estimates in the Exponential Family. Journal of the American Statistical Association. 71 (353): 169 171.doi:10.1080/01621459.1976.10481508. 